Ökobilanzen und umweltbezogene Entlastungseffekte

\section{Eine Bilanz der Bilanz}

\section{Ökobilanzen für Produkte (1) sind in den letzten Jahren zu einem weitgehend akzeptierten Instrumentarium des Umweltmanagements von Unternehmen avanciert (2). Sie helfen, die ökologischen Schwachstellen von Produkten zu identifizieren, und leisten einen Beitrag zum umweltbezogenen Innovationsma- nagement. Doch hat ihre mittlerweile weit verbreitete Anwendung tatsächlich ökologische Entlastungseffekte zur Folge?}

I

Von Gerd Scholl und Susanne Nisius Verkehr des Landes Baden-Württemberg im Rahmen des Projekts Angewandte Ökologie (PAÖ) geförderten Forschungsvorhaben ist das IÖW der in der Einleitung gestellten Frage anhand von acht Fallbeispielen nachgegangen (3). Im folgenden wollen wir beispielhaft einen Anwendungsfall skizzieren, darauf aufbauend das Innovationspotential von Ökobilanzen diskutieren und zuletzt einige Schlußfolgerungen formulieren.

\section{Ökobilanz eines Staubsaugerrohrs}

Die AEG Hausgeräte GmbH fertigt verschiedene Elektro-Klein- und Großgeräte, wie Waschmaschinen, Kühlschränke und Staubsauger. Das Unternehmen zählt in der Branche zu den ÖkoPionieren. Seit den späten achtziger Jahren setzt es auf umfangreiche Maßnahmen zum unternehmens- und produktbezogenen Umweltschutz. Die Ökobilanz des Staubsaugerrohrs wurde 1992 vom Forschungszentrum DaimlerBenz in Ulm in Zusammenarbeit mit dem Unternehmen, das seinerzeit noch zum Daimler-BenzKonzern gehörte, erstellt. Die Bilanzierung der beiden Alternativen verchromtes Stahlrohr und PVC-Rohr stellte ein Pilotprojekt dar, welches ökologische Schwachstellen aufzeigen und zur „Versachlichung“ der Diskussion um den Werkstoff PVC beitragen sollte. Die Ökobilanz bezog sich lediglich auf die Fertigungsschritte der beiden Rohre, vor- und nachgelagerte Stufen des Lebenszyklus wurden ausgeklammert.

In der Gegenüberstellung der Staubsaugerrohre aus PVC und verchromtem Stahl kommt die Ökobilanz - wahrscheinlich nicht zuletzt aufgrund des eingeschränkten Bilanzraumes - zu keinem eindeutigen Ergebnis zugunsten eines der beiden Rohre. Es zeigt sich in der Sachbilanz, daß das PVC-Rohr bezïglich des Energieeinsatzes besser abschneidet als das Stahlrohr. Der vergleichsweise große Energiebedarf für die Stahlproduktion ist auch die Ursache dafür, daß die Luftemissionen (Kohlenmonoxid, Kohlendioxid und Schwefeldioxid) höher sind als die entsprechenden Werte für das PVC-Rohr. Auch auf der Ebene der Wirkungsabschätzung ist das PVC-Rohr dem Stahlrohr in fast allen Wirkungskategorien überlegen (Treibhauseffekt, Versauerung, Eutrophierung, Bildung von Photooxidantien, Humantoxizität und chemische Abfälle). Lediglich hinsichtlich der Ökotoxizität wird das PVC-Rohr wesentlich schlechter als das Stahlrohr beurteilt. Die Studie schließt daher mit der Schlußfolgerung, daß die Bilanz ,nicht als eindeutige Entscheidungsunterstïtzung “ für die Entscheidung zwischen den beiden Alternativen herangezogen werden könne. Da die Ökobilanz zu keinen Veränderungsmaßnahmen geführt hat, sind von ihr keine direkten ökologischen Entlastungseffekte ausgegangen.

Aus heutiger Sicht der AEG Hausgeräte wird die damalige Pilotstudie als ,orientierende Ökobilanz" bezeichnet, die einen Lernprozeß in Gang gesetzt hat. Sie markiert den Anfang einer Reihe von weiteren Ökobilanzen im Unternehmen. Im Falle einer vergleichenden Ökobilanz von Kunststoffschalen für Bodenstaubsauger beispielsweise kam die Untersuchung zu dem eindeutigen Ergebnis, daß die Umweltbelastung mit steigendem Recyclatanteil abnimmt. Aufgrund dieses Ergebnisses werden die Bodenschalen seither nur noch aus recyceltem Kunststoff hergestellt, d. h. hiermit auch Umweltentlastungen erzielt. Ökobilanzen können also ein indirekter Stimulus für positive Umwelteffekte sein.

Der Waschmittelproduzent Henkel kam im Rahmen einer Ökobilanz zu dem Ergebnis, daß die vollständige Substitution eines petrochemi- schen, d. h. auf Erdölbasis hergestellten Tensids, durch eine oleochemische Alternative auf Palmölbasis eine Einsparung von ca. 30 Prozent Erdöl pro Kilogramm Waschmittel ermöglicht. Aufgrund von Qualitätsbedenken (Löslichkeit des Waschmittels) und ökonomischen Erwägungen (höherer Preis der umweltverträglicheren Alternative) wurde die Umstellung allerdings nie vollständig realisiert.

Die Neumarkter Lammsbräu, Marktfïhrer in Sachen Ökobier, bietet seit 1995 ausschließlich Ökoprodukte an. Die vollständige Umstellung auf Rohstoffe aus kontrolliert biologischem Anbau, die aufgrund von Empfehlungen von Ökobilanzen seit Anfang der neunziger Jahre konsequent verfolgt wurde, hat durch die umweltschonenderen Anbaumethoden (z.B. Wegfall der Produktion von Kunstdünger) $\mathrm{zu}$ einer Abnahme der spezifischen $\mathrm{CO}_{2}$-Emissionen um ca. 60 Prozent und zu verringerten Cadmium- und Nitratwerten im Endprodukt gefuihrt.

\section{Das Innovationspotential von Ökobilanzen}

Wie die obigen Beispiele zeigen, sind ökologische Entlastungseffekte durch Ökobilanzen das Ergebnis eines dynamischen Anpassungsprozesses. Ablauf und Ergebnis dieses Prozesses werden von verschiedenen Faktoren beeinflußt:

- So ist das ökologische Innovationspotential zunächst von der Art der jeweiligen Umweltbelastung abhängig. Die Bilanz des Waschmitteltensids hat z.B. Schwachstellen im Bereich der Luftemissionen ausgemacht, die bei vorgelagerten, energieintensiven Stufen des Lebenszyklus auf die Art der Energiebereitstellung (Kohle, Gas, Kernenergie etc.) zurückzuführen sind. Hierauf hat Henkel aber nur sehr geringen Einfluß. In diesem Falle entpuppen sich die tatsächlichen Entlastungsmöglichkeiten also als minimal, weil die Steuerungsmöglichkeiten des Unternehmens beschränkt sind.

- Ein weiterer Punkt betrifft die technische Reife eines Produktes. Je ausgereifter das Produkt, desto geringer fällt das technische Optimierungspotential aus, d. h. mögliche Änderungen beziehen sich dann eher auf die Fertigungstechnologie oder die Distributionslogistik.

- In ähnlicher Weise wirkt sich die Marktreife eines Produktes auf die ökologischen Gestaltungsspielräume aus. Sie beschreibt einen Teil des ökonomischen Lebenszyklus, der mit der Markteinführung beginnt, über eine Wachs-tumsphase die Marktreife erreicht, um an- 
schließend in eine Abschwungphase einzutreten (4). Während der Marktreife eines Produkts ergeben sich aufgrund des Mengenmaximums die größten ökologischen Entlastungspotentiale. Andererseits fällt hier aber auch die Umstellungsflexibilität des Unternehmens am geringsten aus - erfolgreiche Produkte werden, wenn überhaupt, dann nur geringfïgig verändert.

- Auch die Art des Produktes hat Einfluß auf den ökologischen Anpassungsprozeß. Die Mehrheit der von uns untersuchten Ökobilanzen hatte unterschiedlich komplexe Konsumgüter zum Gegenstand (Staubsauger, Teppichböden, Bürostïhle, Waschmittel, Bier). Hier agieren die Unternehmen direkt auf Endverbrauchermärkten, auf denen ökologische Produktqualitäten einen höheren Stellenwert haben als auf Märkten für Zwischen- und Hilfsprodukte ( $\mathrm{z}$. B. Zwirn, Verpackungen). Dies wirkt sich ceteris paribus positiv auf den Umsetzungsprozeß von Bilanzergebnissen aus.

\section{- SchluBfolgerungen und Ausblick}

Die Erwartung, daß Ökobilanzen für Produkte zu ökologischen Verbesserungen bzw. Entlastungen führen, läßt sich bisher nur vorsichtig verifizieren. Um Entlastungspotentiale zu nutzen, müssen praktische Umsetzungsbarrieren identifiziert und überwunden werden. Dies kann beispielsweise durch interne Fortbildungsmaßnahmen, den Aufbau von geeigneter Bilanzierungssoftware mit integrierten Datenbanken oder die Intensivierung des Dialogs mit vor- und nachgelagerten Akteuren geschehen.

Da Ökobilanzen in der Praxis selten dem ,theoretischen Idealmodell“ im Sinne der Normendiskussion entsprechen, sollten methodische Mindeststandards für die Verschlankung von Ökobilanzen (,streamlined LCA“) formuliert werden. Diese können sich z. B. auf eine Wahrung der Transparenz und Nachvollziehbarkeit der Bilanzen beziehen (5).

Produktbezogene Ökobilanzen sollten stärker in betriebliche Entscheidungsprozesse eingebettet werden. Hier kommen z. B. eine Dynamisierung im Sinne einer kontinuierlichen Datenpflege und eines Vergleichsprozesses im Zeitablauf und die Bildung von Produktkennzahlen in Frage. Auch bei der Revision der Öko-Audit Verordnung im Jahre 1998 sollte die Funktion von produktbezogenen Ökobilanzen zur Dokumentation und Förderung des kontinuierlichen Verbesserungsprozesses geprüft werden. Ein Praxis-Leitfaden zur ,Anwendung und Um- setzung von produktbezogenen Ökobilanzen", in dem Unternehmen konkrete Hilfestellung finden, wie sie die Nutzung des Instruments Ökobilanzierung optimieren können, wird in einem Folgeprojekt vom IÖW erstellt. Zu diesem Zweck werden die identifizierten hemmenden und fördernden Faktoren für die Anwendung von Ökobilanzen nochmals evaluiert, mit Experten diskutiert und daraus Hinweise abgeleitet, die Praktikern in Unternehmen die Umsetzung von Ökobilanzergebnissen erleichtern sollen. Der Leitfaden wird voraussichtlich ab Frühjahr 1998 verfügbar sein.

\section{Anmerkungen}

(1) Der Begriff "Ökobilanzen für Produkte“ entspricht der Normvorgabe durch DIN ISO 14040. Wir verwenden synonym die Begriffe "Produktbilanz", "Produkt-Ökobilanz", "produktbezogene Ökobilanz" etc.. Vgl. DIN <Deutsches Institut für Normung> (1994): Grundsätze produktbezogener Ökobilanzen. German „Memorandum of Understanding" / "Conceptual framework" sowie ISO/DIS 14040 „Environmental management - Life cycle assessment - Principles and framework."

(2) Rubik, Frieder (1996): „Themen, Initiatoren, Methoden, Summaries und Anwendungen - Wohin bewegen sich Produkt-Ökobilanzen?", in: UTECH Berlin '97: Produktbezogene Ökobilanzen V, Berlin 19. und 20.2.1997, S. 29-50. (3) Die Fallbeispiele waren AEG Hausgeräte (Staubsaugerrohr), Augsburger Kammgarn Spinnerei (Zwirn), Bosch-Junkers (Verpackungen), Byk Gulden (Verpackungen), Donau Tufting (Teppiche), Grammer (Bürostühle), Henkel (Waschmitteltenside) und Neumarkter Lammsbräu (Bier). Sie sind als Zusammenfassung im Endbericht des Forschungsvorhabens enthalten, vgl. Rubik, Frieder; Grotz, Susanne; Scholl, Gerd (1997): Ökologische Entlastungseffekte durch Produktbilanzen. Karlsruhe: Landesanstalt für Umweltschutz (ISBN 3-88251-244-X). Sechs der acht Fallstudien sind als Schriftenreihe Nr. 115/97 beim IÖW erhältlich.

(4) Kotler, Philip (1991): Marketing Management. Analysis, Planning, Implementation and Control. Seventh Ed., New York et al.: Englewood Cliffs

(5) Vgl. zur Diskussion um "streamlining" z.B. Weitz, Keith A.; Todd, Joel A.; Curran, Mary A.; Malkin, Melissa J. (1996): „Streamlining Life Cycle Assessment. Considerations and a Report on the State of Practice." In: Int.J.LCA, Vol.1, Nr.2, S. 79-85.

\section{Die Autorlmnen}

Gerd Scholl, Dipl. Volkswirt, und Susanne Nisius, Dipl. Kauffrau, beide tätig als wissenschaffliche MitarbeiterInnen im Forschungsfeld "Ökologische Produktpolitik" des IÖW, Heidelberg

Kontakt: IÖW Regionalbüro-Baden-Württemberg, Bergheimersir. 95, 69115 Heidelberg, Tel. 06221/167954, Fax 06221/27060, Email: mailbox@ioew.hd.eunet.de

\section{Zum Thema...}

Sechsmal im Jahr: Die Fachzeitschrift für Umweltrecht und damit sechsmal im Jahr ein kompletter Überblick über das Umweltrecht.

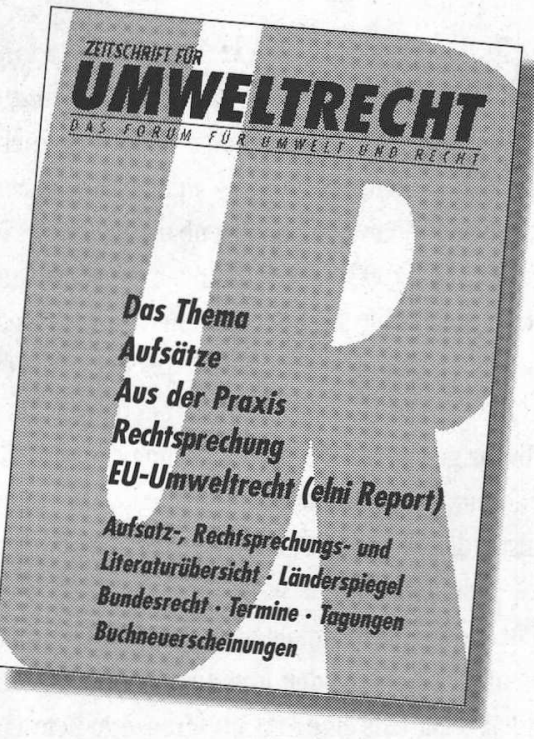

\section{Aktuelle wissenschaftliche Beiträge und Analysen}

- diskutieren Stand und Entwicklung des Umweltrechts.

\section{Ein umfangreicher Service-Teil}

- bringt die neueste Rechtsprechung,

- informiert über die aktuelle Gesetzesentwicklung auf Landes-, Bundes- und Europaebene und

- dokumentiert Aufsätze aus über einhundert Fachzeitschriften sowie wichtige Nachrichten, Termine und Buchneverscheinungen.

Zu bestellen bei: Rhombos-Verlag Kurfürstenstr. 17, 10785 Berlin Tel. 030/2619461, Fax 030/2616854 
(c) 20I0 Authors; licensee IÖW and oekom verlag. This is an article distributed under the terms of the Creative Commons Attribution Non-Commercial No Derivates License (http://creativecommons.org/licenses/by-nc-nd/3.o/), which permits unrestricted use, distribution, and reproduction in any medium, provided the original work is properly cited. 